\title{
Identification of miRNAs and their targets by high-throughput sequencing and degradome analysis in cytoplasmic male-sterile line NJCMS1A and its maintainer NJCMS1B of soybean
}

Xianlong Ding, Jiajia Li, Hao Zhang, Tingting He, Shaohuai Han, Yanwei Li, Shouping Yang ${ }^{*}$ and Junyi Gai

\begin{abstract}
Background: Cytoplasmic male sterility (CMS) provides crucial breeding materials that facilitate hybrid seed production in various crops, and thus plays an important role in the study of hybrid vigor (heterosis), in plants. However, the CMS regulatory network in soybean remains unclear. MicroRNAs (miRNAs) play crucial roles in flower and pollen development by targeting genes that regulate their expression in plants. To identify the miRNAs and their targets that exist in the soybean CMS line NJCMS1A and its maintainer NJCMS1B, high-throughput sequencing and degradome analysis were conducted in this study.

Results: Two small RNA libraries were constructed from the flower buds of the soybean CMS line NJCMS1A and its maintainer NJCMS1B. A total of 105 new miRNAs present on the other arm of known pre-miRNAs, 23 new miRNA members, 158 novel miRNAs and 160 high-confidence soybean miRNAs were identified using high-throughput sequencing. Among the identified miRNAs, 101 differentially expressed miRNAs with greater than two-fold changes between NJCMS1A and NJCMS1B were discovered. The different expression levels of selected miRNAs were confirmed by stem-loop quantitative real-time PCR. A degradome analysis showed that 856 targets were predicted to be targeted by 296 miRNAs, including a squamosa promoter-binding proteinlike transcription factor family protein, a pentatricopeptide repeat-containing protein, and an auxin response factor, which were previously shown to be involved in floral organ or anther development in plants. Additionally, some targets, including a MADS-box transcription factor, NADP-dependent isocitrate dehydrogenase and $\mathrm{NADH}$-ubiquinone oxidoreductase $24 \mathrm{kDa}$ subunit, were identified, and they may have some relationship with the programmed cell death, reactive oxygen species accumulation and energy deficiencies, which might lead to soybean male sterility.
\end{abstract}

Conclusions: The present study is the first to use deep sequencing technology to identify miRNAs and their targets in the flower buds of the soybean CMS line NJCMS1A and its maintainer NJCMS1B. The results revealed that the miRNAs might participate in flower and pollen development, which could facilitate our understanding of the molecular mechanisms behind CMS in soybean.

Keywords: Soybean (Glycine max (L.) Merr.), Cytoplasmic male sterility, MicroRNA, High-throughput sequencing, Degradome analysis

\footnotetext{
* Correspondence: spyung@126.com; sri@njau.edu.cn

Soybean Research Institute, National Center for Soybean Improvement, MOA

Key Laboratory of Biology and Genetic Improvement of Soybean (General),

State Key Laboratory of Crop Genetics and Germplasm Enhancement,

Nanjing Agricultural University, Nanjing, Jiangsu 210095, China
}

\section{) Biomed Central}

(c) 2016 Ding et al. Open Access This article is distributed under the terms of the Creative Commons Attribution 4.0 International License (http://creativecommons.org/licenses/by/4.0/, which permits unrestricted use, distribution, and reproduction in any medium, provided you give appropriate credit to the original author(s) and the source, provide a link to the Creative Commons license, and indicate if changes were made. The Creative Commons Public Domain Dedication waiver (http://creativecommons.org/publicdomain/zero/1.0/) applies to the data made available in this article, unless otherwise stated. 


\section{Background}

In higher plants, cytoplasmic male sterility (CMS) is a natural phenomenon resulting from maternal inheritance, mainly by mitochondrial genes and nuclear gene interactions, which lead to pollen abortion but normal pistil development [1-3]. At present, 200 species of plants are known to exhibit CMS [4], which has been widely used in crops, especially in rice heterosis utilization and crop breeding. Many studies have reported that CMS-related genes are located in the mitochondrial genome, and that its $\mathrm{F}_{1}$ hybrid fertility could be recovered by a restorer gene or genes originating in the nuclear genome [5]. In 1985, CMS in soybean (Glycine $\max (\mathrm{L}$.) Merr.) was first reported by Davis [6], and further characterized by Sun et al. [7]. At present, a variety of soybean CMS lines, such as NJCMS1A $[8,9]$ and NJCMS2A [10], have been developed. With the construction of three lines, CMS, maintainer and restorer, of soybean, many soybean hybrids have been successfully cultivated.

MicroRNAs (miRNAs) are a class of small, endogenous 21 -nt non-coding RNAs. In plants, they are processed from single-stranded RNA precursors that can form stem-loop regions $[11,12]$. These miRNAs play a critical role in nearly all biological processes: development, differentiation, and biotic and abiotic stress responses in higher plants, via the degradation or translational inhibition of target mRNAs at transcriptional and post-transcriptional levels [13]. miRNAs have been discovered using three basic approaches: direct cloning, forward genetics and bioinformatics predictions with experimental validation [11]. In recent years, degradome sequencing has been used to identify global targets of miRNAs in Arabidopsis thaliana and soybean [14, 15].

To date, hundreds of miRNAs have been isolated by direct cloning or deep sequencing in soybean [16-22], but the number of miRNAs known in soybean is still very small and considerably lower than in Arabidopsis thaliana or rice. Additionally, in the past several years, the miRNAs associated with CMS, genetic male sterility or male sterility in plants have been studied, and the results showed that the miRNAs helped regulate male sterility and fertility restoration [23-28]. However, as a widely planted oil crop worldwide, the roles of miRNAs in soybean CMS remain largely unknown. Thus, the identification of miRNAs and the elucidation of their functions in pollen development will help us understand the molecular mechanisms of soybean CMS. Here, we examined and compared the expression profiles of miRNAs in the flower buds of a soybean CMS line NJCMS1A and its maintainer NJCMS1B, using highthroughput sequencing. We also applied degradome sequencing to identify genes targeted by identified
miRNAs and analyzed their possible functions. The results may indicate that there were some interactions between miRNAs and their targets, therefore flower bud or pollen development could be influenced by the biogenesis of miRNAs in CMS.

\section{Results}

Analysis of small RNA library data sets from the flower buds of NJCMS1A and NJCMS1B

Two independent small RNA libraries were constructed using RNAs from the flower buds of CMS line NJCMS1A and its maintainer NJCMS1B. A total of 12 104688 and 12096511 raw reads from the two lines, respectively, were generated by high-throughput sequencing (Table 1). After the removal of adaptor contaminants, oversized insertions, low-quality reads, poly A tags and small tags < 18-nt, 11974839 and 12058427 clean reads, respectively, were obtained with lengths that range from 18-30-nt, and the mappable small RNA sequences were 9824678 and 9758 494, respectively (Table 1). Then, the datasets were used to remove other non-coding RNAs and the degraded fragments of mRNAs (Additional file 1: Table S1). The majority of the small RNAs in the two libraries were 21-24 nt, and the most abundant small RNAs were $24 \mathrm{nt}$, followed by 21 and 22 nts (Fig. 1), which is within the typical small RNA length distribution for soybean tissues, such as roots, nodules, flowers, developing seeds and cotyledons $[19,22,29]$.

\section{Identification of known miRNAs}

All mappable small RNA sequences were compared with the known soybean miRNAs in the miRBase database (miRBase 21.0, http://www.mirbase.org/). In miRBase 21.0, 573 soybean pre-miRNAs correspond to 639 mature miRNAs. In the present study, 503 pre-miRNAs corresponding to 560 mature miRNAs, were detected that belonged to 222 families (Additional file 1: Table $\mathrm{S} 2$ ). The length of most known miRNAs was $21 \mathrm{nt}$, followed by 24 and 22 nts (Additional file 2: Figure S1). Among these miRNA families, MIR156 had 24 members, followed by MIR166 with 21 members, but most families had fewer than 10 members (Additional file 1: Table S3, Additional file 2: Figure S2). Interestingly, numerous miRNAs, having additional nucleotides in the $5^{\prime}$ or 3' termini when compared with the recorded mature miRNAs in miRBase, were detected and could be classified as isomiRNAs. This type of miRNA was previously reported in soybean after high-throughput sequencing [30]. A total of 205 isomiRNAs were discovered in our study and their respective mature miRNAs can be found in Additional file 1: Table S2, with "D" tags. 
Table 1 Analysis of small RNA sequences and degradome sequences from NJCMS1A and NJCMS1B

\begin{tabular}{|c|c|c|c|c|c|c|}
\hline \multirow[t]{2}{*}{ Type } & & & \multicolumn{2}{|c|}{ small RNA-Seq } & \multicolumn{2}{|c|}{ Degradome-Seq } \\
\hline & & & NJCMS 1A & NJCMS 1B & NJCMS $1 \mathrm{~A}$ & NJCMS 1B \\
\hline \multirow[t]{2}{*}{ Statistical Information } & Total tags & & $\begin{array}{l}12104688 \\
(100.00 \%)\end{array}$ & $\begin{array}{l}12096551 \\
(100.00 \%)\end{array}$ & $\begin{array}{l}12351703 \\
(100.00 \%)\end{array}$ & $\begin{array}{l}12671179 \\
(100.00 \%)\end{array}$ \\
\hline & Clean tags & & $\begin{array}{l}11974839 \\
(98.93 \%)\end{array}$ & $\begin{array}{l}12058427 \\
(99.68 \%)\end{array}$ & $\begin{array}{l}12288476 \\
(99.49 \%)\end{array}$ & $\begin{array}{l}12613736 \\
(99.55 \%)\end{array}$ \\
\hline \multirow[t]{4}{*}{$\begin{array}{l}\text { Genome Mapping } \\
\text { Statistics }\end{array}$} & $\begin{array}{l}\text { Total Count of Clean } \\
\text { Tags }\end{array}$ & Total tags & $\begin{array}{l}11974839 \\
(100.00 \%)\end{array}$ & $\begin{array}{l}12058427 \\
(100.00 \%)\end{array}$ & $\begin{array}{l}12288476 \\
(100.00 \%)\end{array}$ & $\begin{array}{l}12613736 \\
(100.00 \%)\end{array}$ \\
\hline & & $\begin{array}{l}\text { Mapping to } \\
\text { genome }\end{array}$ & $\begin{array}{l}9824678 \\
(82.04 \%)\end{array}$ & $\begin{array}{l}9758494 \\
(80.93 \%)\end{array}$ & $\begin{array}{l}10954689 \\
(89.15 \%)\end{array}$ & $\begin{array}{l}11289967 \\
(89.51 \%)\end{array}$ \\
\hline & $\begin{array}{l}\text { Unique Count of Clean } \\
\text { Tags }\end{array}$ & Total tags & $\begin{array}{l}3668753 \\
(100.00 \%)\end{array}$ & $\begin{array}{l}4122100 \\
(100.00 \%)\end{array}$ & $\begin{array}{l}6332697 \\
(100.00 \%)\end{array}$ & $\begin{array}{l}5445882 \\
(100.00 \%)\end{array}$ \\
\hline & & $\begin{array}{l}\text { Mapping to } \\
\text { genome }\end{array}$ & $\begin{array}{l}2687857 \\
(73.26 \%)\end{array}$ & $\begin{array}{l}3142047 \\
(76.22 \%)\end{array}$ & $\begin{array}{l}5500905 \\
(86.87 \%)\end{array}$ & $\begin{array}{l}4663560 \\
(85.63 \%)\end{array}$ \\
\hline
\end{tabular}

\section{Conservation of known miRNAs}

Most miRNAs (52.68 \%) are conserved among different plant species, and play important and conserved functions in plant development, including flower and pollen development. In contrast, non-conserved miRNAs may play roles in more species-specific characteristics in plant development [31]. In the present study, the known miRNAs belonged to 222 families and 43 of them were conserved in other species (including 12 leguminousspecific miRNA families), while the rest were nonconserved soybean-specific miRNA families (Table 2). Zhang et al. [31] demonstrated that the miRNAs found in more than 10 different plant species were considered highly conserved. Similarly, the miRNA families classified as moderately, lowly and non-conserved miRNAs were found in five to nine, two to four, and only one plant species, respectively [31]. In this study, 27 highly conserved, four moderately conserved and 12 lowly conserved miRNA families were found, in addition, there were 179 non-conserved miRNA families (Additional file 1: Table S4).

\section{Novel miRNAs on the other arm of known pre-miRNAs}

Sometimes, the miRNA-3p and miRNA-5p are simultaneously present on the two arms of the pre-miRNA secondary structures, and are considered strong evidence for the existence of these miRNAs in plant. Four criteria described in Methods were used to ensure the accuracy of the results. Through high-throughput sequencing, 105 novel miRNAs on the other arm of known pre-

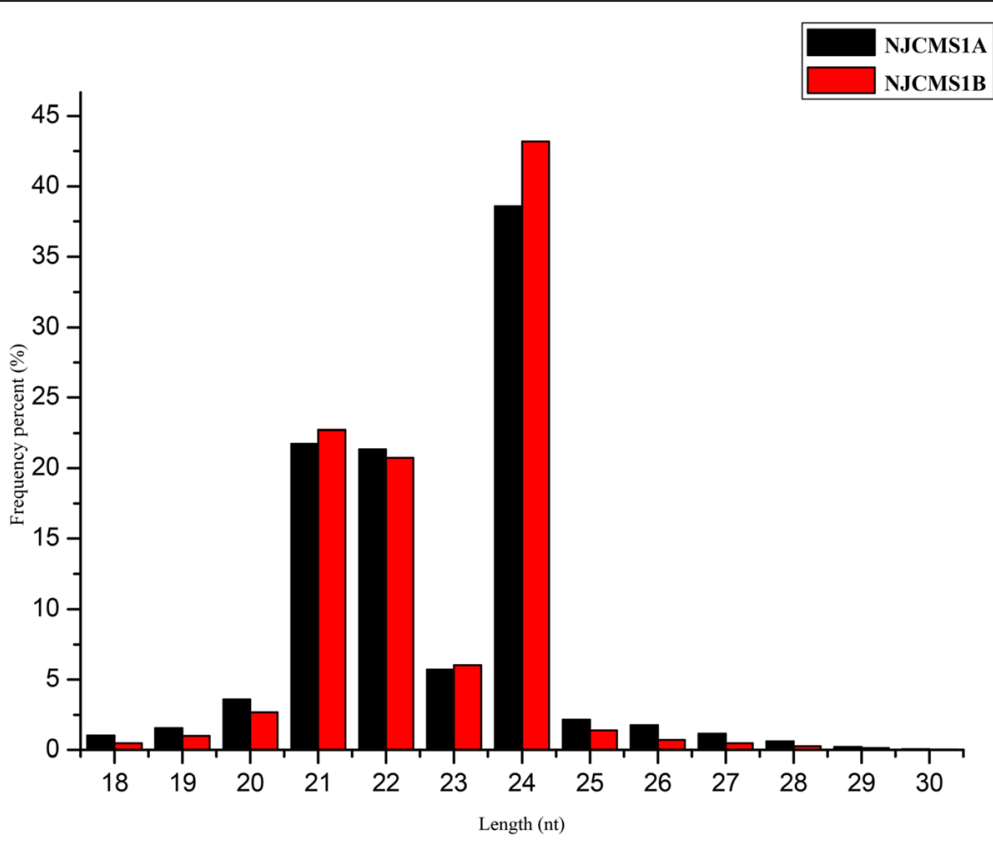

Fig. 1 Length distribution of small RNAs found in flower buds from NJCMS1A and NJCMS1B 
Table 2 Summary of miRNAs, and their families, found in the flower buds of NJCMS1A and NJCMS1B

\begin{tabular}{|c|c|c|c|c|c|}
\hline \multirow[t]{2}{*}{ Type } & \multicolumn{2}{|c|}{ Conserved miRNAs } & \multicolumn{2}{|l|}{ Non-conserved miRNAs } & \multirow[t]{2}{*}{ Total } \\
\hline & $\begin{array}{l}\text { Found in other } \\
\text { plant species }\end{array}$ & $\begin{array}{l}\text { Only found in } \\
\text { legume species }\end{array}$ & $\begin{array}{l}\text { With miRNA family information } \\
\text { in miRBase } 21.0\end{array}$ & $\begin{array}{l}\text { With no miRNA family information } \\
\text { in miRBase } 21.0\end{array}$ & \\
\hline No. of known miRNAs & 262 & 33 & 108 & 157 & 560 \\
\hline $\begin{array}{l}\text { No. of novel miRNA-3p/ } \\
\text { miRNA-5p }\end{array}$ & 72 & 6 & 15 & 12 & 105 \\
\hline $\begin{array}{l}\text { No. of new miRNAs } \\
\text { members }\end{array}$ & 18 & 0 & 2 & 3 & 23 \\
\hline No. of novel miRNAs & 0 & 0 & 0 & 158 & 158 \\
\hline $\begin{array}{l}\text { No. of known miRNAs } \\
\text { families }\end{array}$ & 31 & 12 & 36 & 143 & 222 \\
\hline $\begin{array}{l}\text { No. of novel miRNA-3p/ } \\
\text { miRNA-5p families }\end{array}$ & 21 & 4 & 8 & 12 & 45 \\
\hline $\begin{array}{l}\text { No. of new miRNAs } \\
\text { members families }\end{array}$ & 5 & 0 & 1 & 3 & 9 \\
\hline $\begin{array}{l}\text { No. of novel miRNAs } \\
\text { families }\end{array}$ & 0 & 0 & 0 & 111 & 111 \\
\hline
\end{tabular}

$\mathbf{a}$

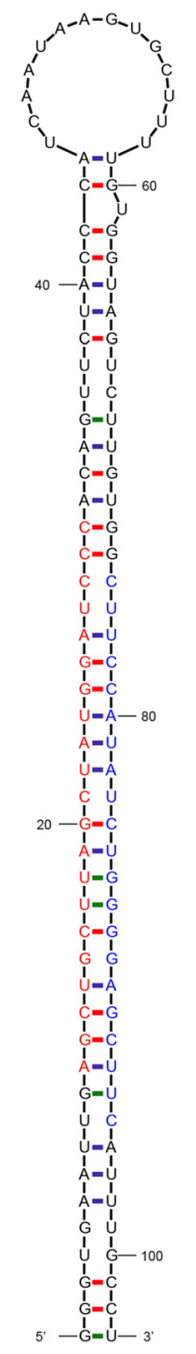

b

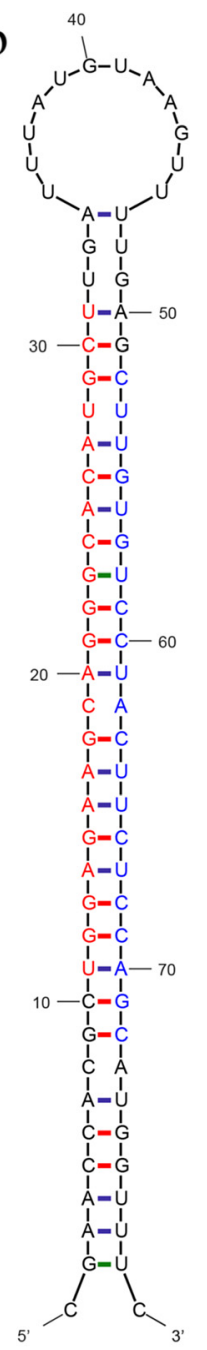

C

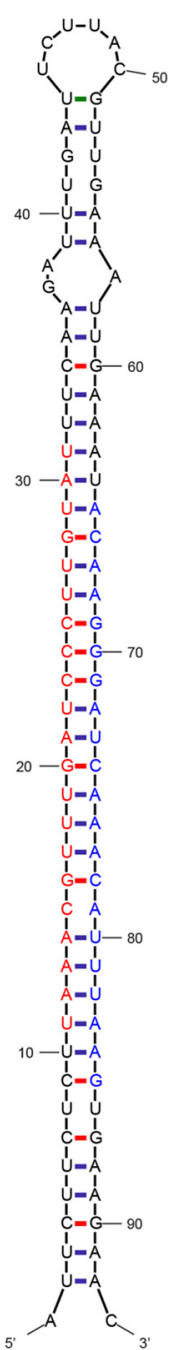

Fig. 2 Predicted secondary structures of novel miRNAs from flower buds of NJCMS1A and NJCMS1B. a gma-miR159d; b N-gma-miR164r; and c novel_mir_051. Red font represents miRNA-5p, and blue font represents miRNA-3p 
miRNAs were identified (Additional file 1: Table S5), which belonged to 45 families and had not been identified previously (Table 2). Five known miRNAs (gmamiR4378a, gma-miR4407, gma-miR4416c, gma-miR9747 and gma-miR9763) were not obtained in this study, but their miRNAs on the other arm of pre-miRNAs were identified, which were named gma-miR4378a-3p, gmamiR4407-5p, gma-miR4416c-3p, gma-miR9747-5p and gma-miR9763-3p, respectively. The predicted secondary structure of gma-miR159d was selected as an example to be shown in Fig. 2a, and the others are shown in Additional file 2: Figure S3.

Identification of newly conserved soybean miRNA families and new miRNA members

All unannotated small RNA sequences that matched soybean genome sequences were mapped to known plant miRNAs in miRBase to identify newly conserved miRNA families and new miRNA members. If the small RNAs matched known plant miRNAs with no more than four mismatches, they were classified as candidate conserved miRNAs and new miRNA members [32]. Four characteristics of miRNAs, described in Methods, were used to screen the candidate conserved miRNAs and new miRNA members [20]. Unfortunately, no new conserved miRNA families were found in the present study. However, 23 new miRNA members, belonging to nine known miRNA families (five conserved and four nonconserved miRNA families; Table 2), were discovered in the two small RNA libraries (Table 3). These 23 miRNAs have not been reported as gma-miRNAs in miRBase before but were homologous to known plant miRNAs. The length of most miRNAs was 21 nt, with only one miRNA having a length of $22 \mathrm{nt}$, which conformed to a common characteristic of plant miRNAs. For N-gmamiR164r, N-gma-miR399i and N-gma-miR399k, both miRNA-3p and miRNA-5p were simultaneously found on the two arms of their pre-miRNAs. Of seven new miRNA members that belonged to MIR164, six shared the same mature sequence, and they had only one base different from gma-miR164r-5p. However, their precursors came from different loci of the soybean genome. A similar result was found for the MIR399 and MIR4380 families (Table 3). The precursor sequences of N-gmamiR164l and N-gma-miR164n, as well as N-gmamiR164m and N-gma-miR164p, were highly similar to

Table 3 Identification of new miRNA members of known miRNA families

\begin{tabular}{|c|c|c|c|c|c|c|c|c|}
\hline Index & miRNA_name & miRNA_sequence & Length (nt) & MFE (kcal/mol/nt) & MFEI & NJCMS1A_count & NJCMS1B_count & $3 p / 5 p^{a)}$ \\
\hline 1 & N-gma-miR164l & TGGAGAAGGGGAGCACGTGCA & 21 & -0.53 & 0.98 & 12 & 8 & $5 p$ \\
\hline 2 & N-gma-miR164m & TGGAGAAGGGGAGCACGTGCA & 21 & -0.52 & 0.97 & 12 & 8 & $5 p$ \\
\hline 3 & N-gma-miR164n & TGGAGAAGGGGAGCACGTGCA & 21 & -0.54 & 0.97 & 12 & 8 & $5 p$ \\
\hline 4 & N-gma-miR164o & TGGAGAAGGGGAGCACGTGCA & 21 & -0.53 & 1.15 & 12 & 8 & $5 p$ \\
\hline 5 & N-gma-miR164p & TGGAGAAGGGGAGCACGTGCA & 21 & -0.48 & 0.90 & 12 & 8 & $5 p$ \\
\hline 6 & N-gma-miR164q & TGGAGAAGGGGAGCACGTGCA & 21 & -0.49 & 1.07 & 12 & 8 & $5 p$ \\
\hline 7 & N-gma-miR164r-5p & TGGAGAAGCAGGGCACATGCT & 21 & -0.57 & 1.20 & 78 & 35 & $5 p$ \\
\hline 8 & N-gma-miR164r-3p & CTTGTGTCCTACTTCTCCAGC & 21 & -0.57 & 1.20 & 4 & 0 & $5 p$ \\
\hline 9 & N-gma-miR156ac & CTGACAGAAGATAGAGAGCAC & 21 & -0.40 & 0.86 & 85 & 69 & $5 p$ \\
\hline 10 & N-gma-miR395n & CTGAAGTGTITGGGGGAGCTT & 21 & -0.41 & 0.91 & 5 & 6 & $3 p$ \\
\hline 11 & N-gma-miR399i-5p & GAGCAATTCTCCTITGGCAGA & 21 & -0.48 & 1.15 & 2 & 0 & $5 p$ \\
\hline 12 & N-gma-miR399i-3p & TGCCAAAGGAGAATTGTCCTG & 21 & -0.48 & 1.15 & 6 & 0 & $3 p$ \\
\hline 13 & N-gma-miR399j & TGCCAAAGGAGATTTGCCCTG & 21 & -0.44 & 0.98 & 0 & 9 & $3 p$ \\
\hline 14 & N-gma-miR399k-5p & GAGCAAATCTCCATTGGCAGT & 21 & -0.45 & 1.01 & 0 & 5 & $5 p$ \\
\hline 15 & N-gma-miR399k-3p & TGCCAAAGGAGATTTGCCCTG & 21 & -0.45 & 1.01 & 0 & 9 & $3 p$ \\
\hline 16 & N-gma-miR399| & TGCCAAAGGAGAGCTGCCCTG & 21 & -0.43 & 1.07 & 97 & 53 & $3 p$ \\
\hline 17 & N-gma-miR399m & TGCCAAAGGAGAGCTGCCCTG & 21 & -0.45 & 1.17 & 97 & 53 & $3 p$ \\
\hline 18 & N-gma-miR530f & TGCATTGCACCTGCGCTTTG & 21 & -0.47 & 0.96 & 8 & 0 & $5 p$ \\
\hline 19 & N-gma-miR4380c & TGGTTCATACGGATTGTTGAT & 21 & -0.47 & 1.12 & 5 & 0 & $3 p$ \\
\hline 20 & N-gma-miR4380d & TGGTTCATACGGATTGTTGAT & 21 & -0.33 & 1.28 & 5 & 0 & $3 p$ \\
\hline 21 & N-gma-miR4348d & TGTCAAACTTGCAAGATGATA & 21 & -0.45 & 1.46 & 0 & 10 & $5 p$ \\
\hline 22 & N-gma-miR5673b & TGGAATCTCGCGGAAGACATC & 21 & -0.61 & 1.27 & 5 & 0 & $3 p$ \\
\hline 23 & N-gma-miR5786b & CGTCGCAGGATAGAGGGCACTG & 22 & -0.39 & 0.94 & 0 & 8 & $5 p$ \\
\hline
\end{tabular}

$\mathrm{N}$ new. a) Arm of this mature miRNA 
each other (Table 3). These types of miRNAs were termed sub-members [25]. The secondary structures of precursors to N-gma-miR164r were selected as the example to be shown in Fig. 2b, and the others are shown in Additional file 2: Figure S4.

\section{Identification of novel miRNAs}

To predict novel miRNAs in soybean, we used MIREAP (https://source-forge.net/projects/ mireap/) to explore the secondary structures, Dicer cleavage sites and minimum free energies (MFEs) of the unannotated small RNA sequences that could be mapped to the soybean genome. The unannotated small RNAs that map to the soybean genome, but not to known plant miRNAs, were classified as candidate novel miRNAs. Four criteria described in Methods were used to increase the predictive accuracy. A total of 158 new miRNAs, belonging to 111 novel families (Table 2), were identified in this study. Among them, 20 novel miRNAs on both arms of the pre-miRNAs were identified. The lengths of the mature miRNAs ranged from 20 to $23 \mathrm{nt}$, and most of them were $21 \mathrm{nt}$. The precursors of these novel miRNAs were identified by MIREAP and varied from 66 to $354 \mathrm{nt}$ in length, with MFE values ranging from -20.7 to $-181.1 \mathrm{kcal} / \mathrm{mol}(-0.23$ to $-0.86 \mathrm{kcal} / \mathrm{mol} / \mathrm{nt})$. The $\mathrm{min}-$ imal folding energy indices (MFEI) ranged from 0.85 to 2.14, with $85.53 \%$ of them being greater than 0.97 and the average being 1.32 , which is consistent with miRNA. Most of the new mature miRNA sequences (73.58 \%) presented a uracil (U) as the first nucleotide. This is in agreement with previous results for soybean [16]. Like new miRNA members, some of the novel miRNAs, such as novel_mir_018a to novel_mir_018f, shared the same mature sequences but had different precursors that came from different loci. The novel-MIR15 had two submembers (novel_mir_015a and novel_mir_015b). Unlike known miRNAs, the expression levels of the novel miRNAs were very low. The information for all of the novel miRNAs is summarized in Additional file 1: Table S6. The secondary structure for the precursor of novel_mir_51 was used as an example in Fig. 2c, and the others are shown in Additional file 2: Figure S5.

\section{High-confidence miRNAs in soybean}

Until now, no soybean miRNAs had been annotated as high confidence in miRBase 21.0 (http://www.mirbase.org/cgi-bin/mirna_summary.pl?org=gma). To be annotated as a high-confidence miRNA, in addition to the four characteristics of miRNAs described in Methods, a locus must meet the following criteria: first, at least 10 reads map to each of the two mature sequences (miRNA-5p and miRNA-3p), or at least five reads map to each arm and at least 100 reads are mapped in total (miRBase 21.0); and second, at least $50 \%$ of the reads mapping to each arm of the hairpin precursor must have the same $5^{\prime}$ end [33]. Through a combined analysis of known miRNAs with their new 5p or 3p miRNAs, 156 known miRNAs and four novel miRNAs could be annotated as high-confidence miRNAs, representing $23.46 \%$ and $2.53 \%$ of known miRNAs (including new $5 \mathrm{p}$ or $3 \mathrm{p}$ miRNAs) and novel miRNAs in this study, respectively (Additional file 1: Table S7). The secondary structures for precursors of high-confidence miRNAs are shown in Additional file 2: Figure S6.

\section{Differential expression profiling of miRNAs and validation by qRT-PCR}

Among the identified miRNAs, 101 differentially expressed miRNAs with greater than two-fold relative changes $(P<0.05)$ between NJCMS1A and NJCMS1B were identified by high-throughput sequencing. Compared with NJCMS1B, 35 miRNAs were up-regulated with fold changes $>2$ (such as gma-miR1518), and 66 were down-regulated with fold changes $<-2$ (such as gma-miR1512b) in NJCMS1A. Unlike known miRNAs, many novel miRNAs, including novel_mir_014 and novel_mir_039, were only expressed in NJCMS1A or NJCMS1B, respectively. All information regarding the differential expression profiling of miRNAs can be found in Additional file 1: Table S8.

The stem-loop qRT-PCR was conducted to validate and measure the expression profiles of the nine selected differentially expressed miRNAs. As shown in Fig. 3, seven of them were consistent with the sequencing reads. However, novel_mir103 and gma-miR397a were found to be up-regulated in NJCMS1A, but were enriched in NJCMS1B based on the high-throughput sequencing analysis. The trend was not consistent with that of deep sequencing, possibly because of the low expression levels in the two samples and the difference in sensitivity between qRT-PCR and high-throughput sequencing technology.

\section{Identification of miRNA target genes in NJCMS1A and NJCMS1B using a degradome analysis}

Two independent degradome libraries derived from flower buds of NJCMS1A and NJCMS1B were constructed and sequenced. A total of 12351703 and 12 671179 raw reads were generated from the NJCMS1A and NJCMS1B libraries, respectively (Table 1). After removing the adaptor sequences and/or low-quality reads present in the raw reads, 12288476 and 12613736 clean reads in the NJCMS1A and NJCMS1B libraries, respectively, were obtained, of which 10954689 (89.15\%) and 11289967 (89.51\%) were perfectly matched to the soybean genome (Table 1). Moreover, $98.69 \%$ of the clean reads in NJCMS1A and $98.82 \%$ of the clean reads in NJCMS1B were 20 and 21 nts in length, which 


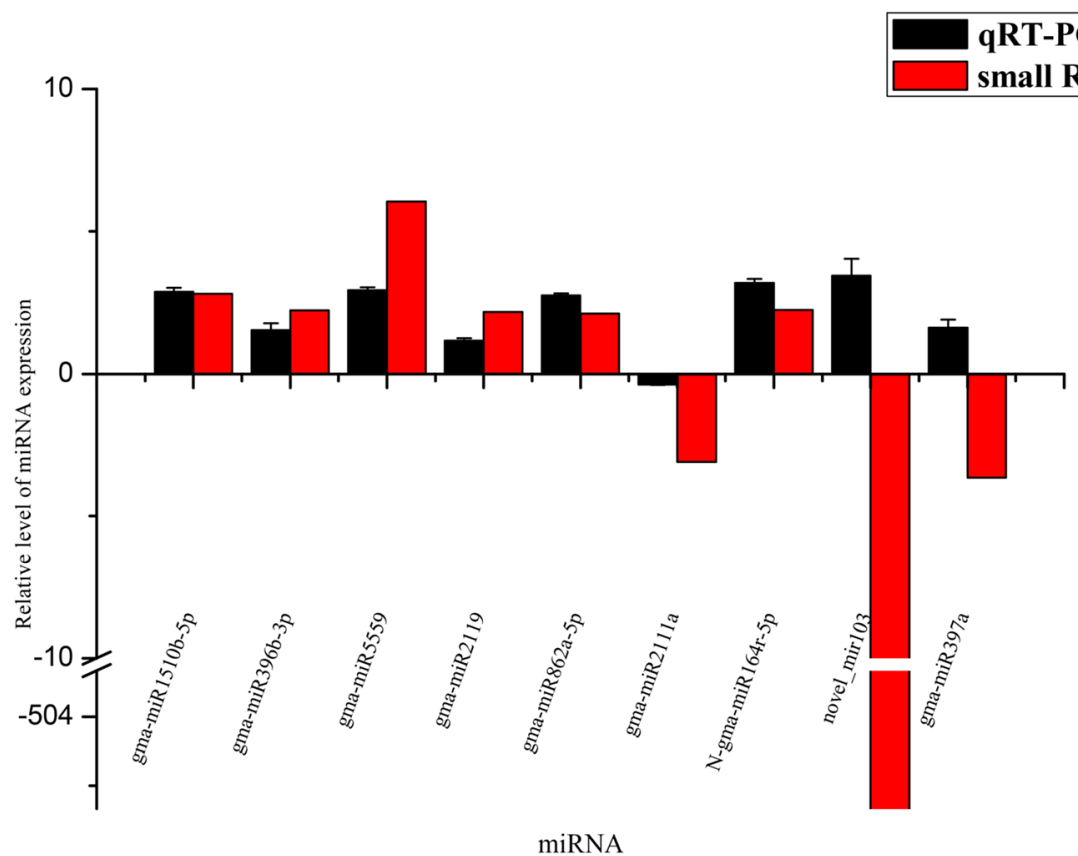

Fig. 3 Expression levels of selected miRNA in NJCMS1A and NJCMS1B. The $y$-axis indicated the miRNA relative expression level generated from qRT-PCR analysis and high-throughput sequencing. The results were obtained from three biological replicates, and the error bars indicated the standard error of the mean of $2^{-\Delta \Delta C t}$, with NJCMS1B as a control

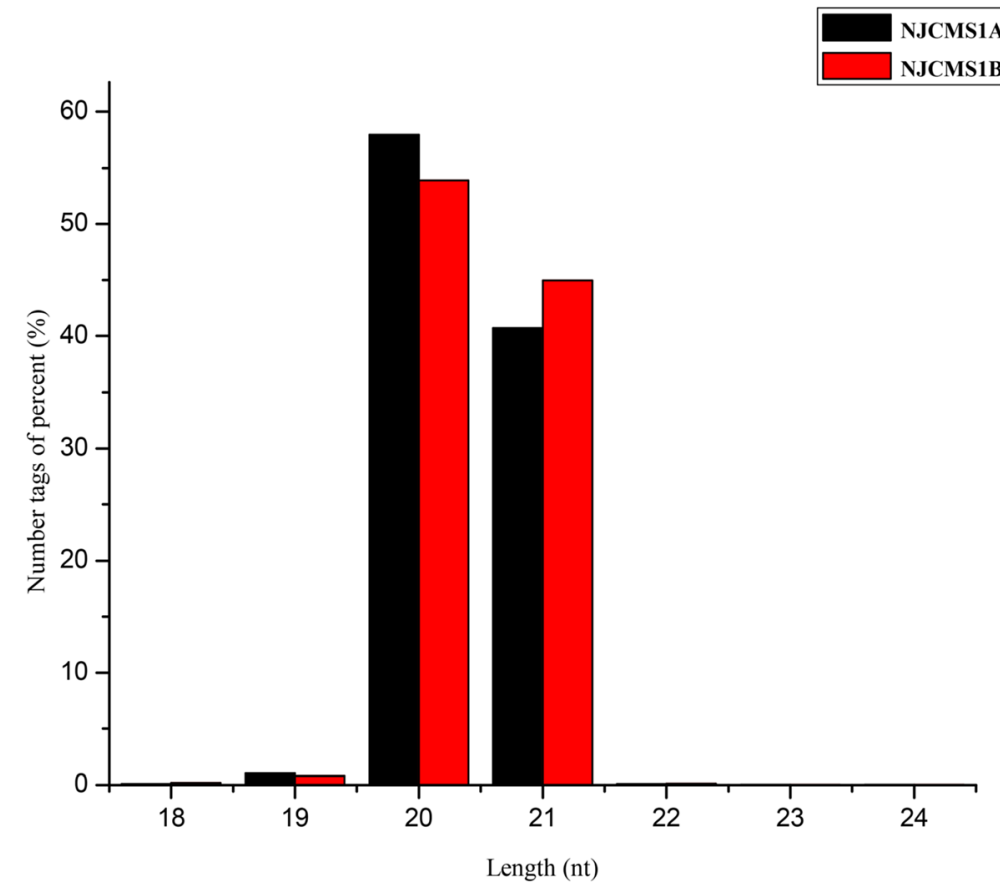

Fig. 4 Length distributions of degradome library clean reads in flower buds of NJCMS1A and NJCMS1B 
matched the length distribution peak of the degradome fragments (Fig. 4).

Based on the distribution of splice sites near raw sequence tags, the splice sites could be divided into five categories: 0 to 4 (Additional file 1: Table S9 and Fig. 5). In total, 856 targets were predicted to be cleaved by 296 miRNAs. Furthermore, 28 novel miRNAs were shown to cleave 81 targets (Additional file 1: Table S10). The targets of all of the miRNAs are listed in Additional file 1: Table S9. These targets included a squamosa promoterbinding protein-like transcription factor family protein, pentatricopeptide repeat-containing (PPR) protein, MADS-box protein, GAMYB protein, auxin response factor, no apical meristem protein, homeobox-leucine zipper protein, nuclear transcription factor Y, transcription factor CCAAT, GRAS family transcription factor, transcription factor APETALA2, TCP transcription factor, F-box family protein, growth-regulating factor, ubiquitin-conjugating enzyme family protein, zinc knuckle (CCHC-type) family protein and basic helixloop-helix DNA-binding superfamily protein, having essential roles in gene regulation. Some of the targets,

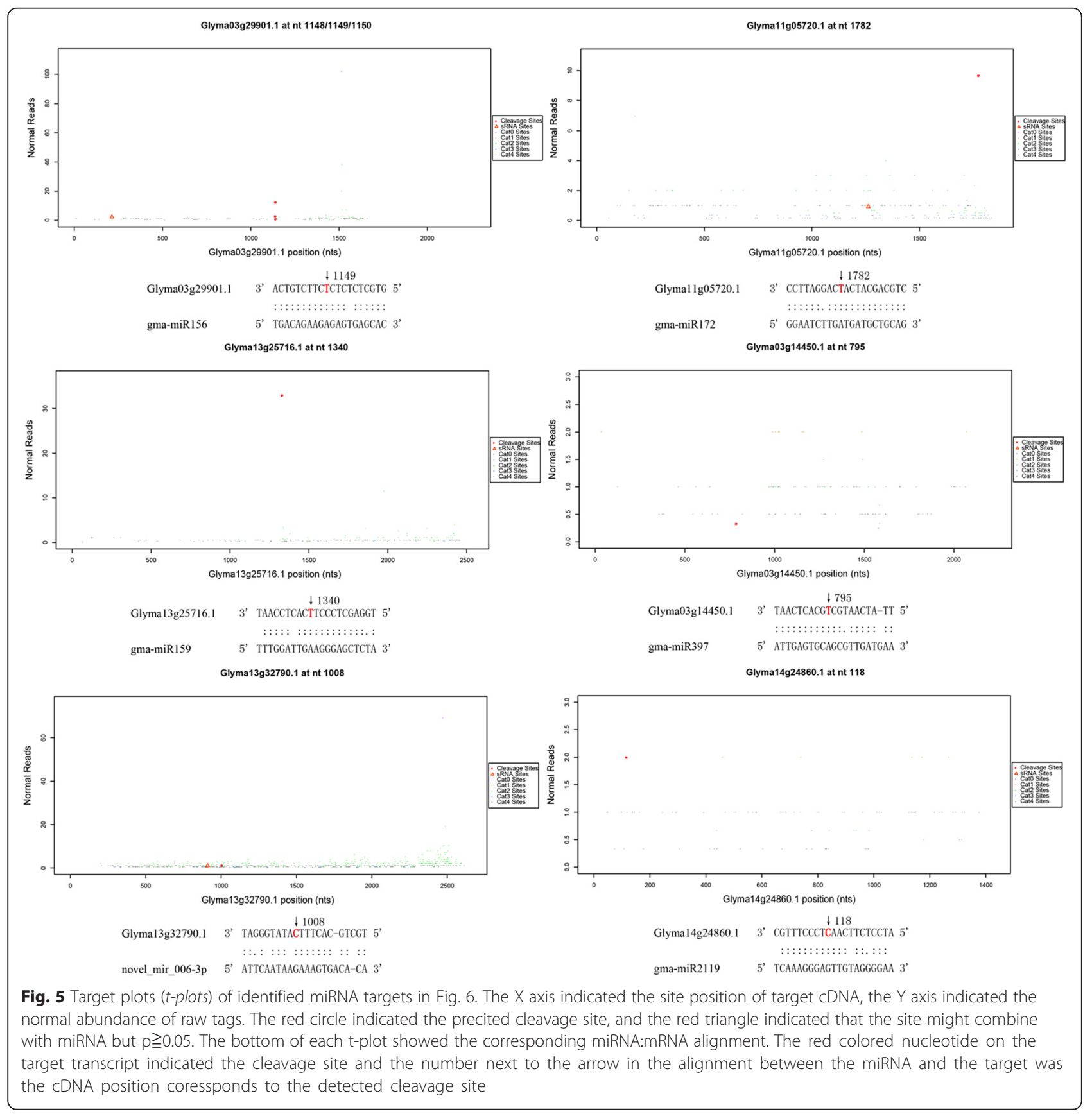


such as laccase (LAC) and heat shock $70 \mathrm{kDa}$ protein (Hsp70) etc., may be related to CMS or pollen development. Unfortunately, the target genes of most identified miRNAs could not be detected in the present degradome analysis. In addition, some target genes have unknown functions (Additional file 1: Table S9).

All of the identified miRNA targets were analyzed by gene ontology (GO, http://www.geneontology.org/) to assess the targets' putative functions and uncover the roles of miRNAs in regulating pollen development. As shown in Additional file 2: Figure S7, the targets were classified into 19 categories in biological processes, followed by 10 and eight categories in cellular components and molecular functions, respectively. Greater percentages of these targets were considered to be involved in metabolic (33.76 \%), cellular (32.01\%), singleorganism (14.02 \%), response to stimulus (12.38\%) and developmental (8.64 \%) processes. Among cellular component categories, cell (32.36 \%) and cell part (32.36 \%) were the main functional groups. For molecular function, the binding (34.58 \%) and catalytic activity $(24.42 \%)$ terms contained the largest numbers of targets. The results may indicate the importance of these miRNAs in gene regulation during pollen development in soybean.

\section{Expression of miRNA targets in NJCMS1A and NJCMS1B}

To test if any inverse relationship between target expression and the level of corresponding miRNA existed, the expression patterns, based on qRT-PCR, of 14 miRNA targets discussed below were studied. As expected, the expression levels of squamosa promoter-binding protein-like, GAMYB, APETALA2, LACs were higher in NJCMS1A than in NJCMS1B, and those of the other 10 targets such as MADS-box protein etc. were lower in NJCMS1A than in NJCMS1B, which were in contrast with their corresponding miRNAs (Fig. 6 and Additional file 2: Figure S8).

\section{Discussion}

Diverse miRNAs and their characteristics in flower buds of NJCMS1A and NJCMS1B

Small RNAs have been identified as pivotal regulators during anther and pollen development in plants [34-36].

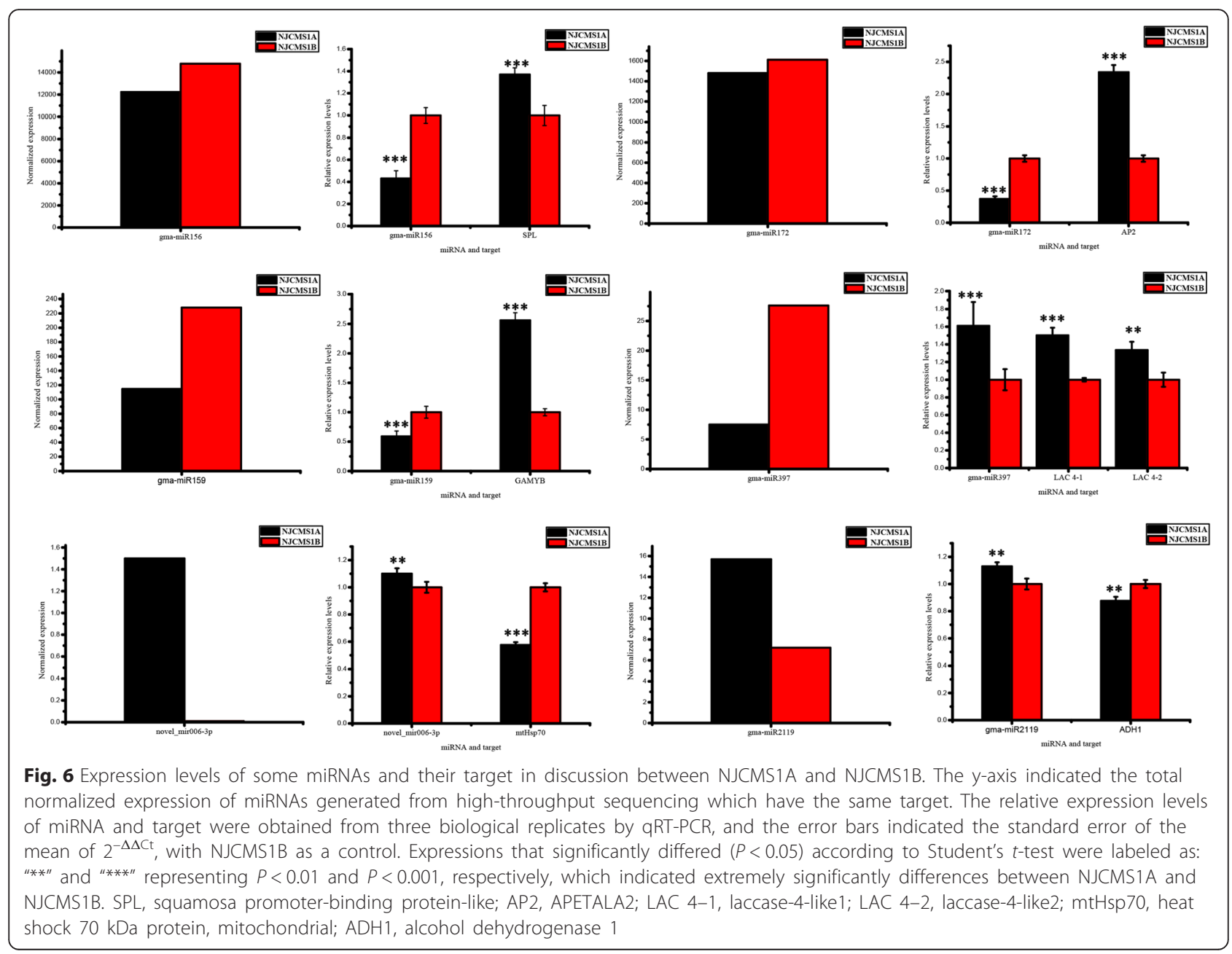


However, no existing studies have reported the relationship between miRNAs and pollen in soybean. To identify miRNAs linked to the regulation of pollen development in soybean, two small RNA libraries were constructed with the flower buds of a CMS line NJCMS1A and its maintainer NJCMS1B. Based on high-throughput sequencing, we identified a much wider range of small RNAs, 18-30 nt in length (Fig. 1), with 24 nt being the most abundant, followed by 21 and then $22 \mathrm{nt}$. The results were consistent with previous reports in various other species, including Arabidopsis thaliana [37], Citrus sativus [38], Medicago truncatula [39], Oryza sativa [40] and Zea mays [41], suggesting that the most abundant small RNA length is 24 nt. Most known miRNAs had the canonical 21 nt length (Additional file 2: Figure S1), suggesting they were Dicer-like1 cleavage products.

The identification of miRNAs has previously been reported in male-sterile plants, including maize, rice, Brassica campestris ssp. chinensis, Brassica juncea and cotton [23-28]. The present study is the first to report the comprehensive identification of miRNAs and their targets using deep sequencing of flower buds between CMS and maintainer lines in soybean. Among the 560 known miRNAs, 205 had additional nucleotides in the $5^{\prime}$ or $3^{\prime}$ terminus, which was caused by an imprecise or alternative cleavage by Dicer during pre-miRNA processing [42]. According to miRBase release 21.0, 135 mature miRNAs (31.62\%, 427 mature sequences in total) were annotated as high confidence in Arabidopsis thaliana, and all of those miRNAs were reported to have their miRNA-5p or miRNA-3p pairing accumulated. However, until now, no miRNAs were annotated as high confidence in soybean. Thus, the identification of novel miRNAs on the other arm of known pre-miRNAs increased the confidence of these miRNAs. With the criteria described by Kozomara et al. [33] and miRBase 21.0, 156 known miRNAs and four novel miRNAs (Additional file 1: Table S7) could be annotated as highconfidence miRNAs.

Considering that some miRNAs are widespread, while others are distributed in limited plant species, all of the known miRNAs have been classified into four categories: "highly conserved", "moderately conserved", "lowly conserved" and "non-conserved". In our study, a high proportion of non-conserved miRNA families (80.63\%) were identified, and only 27 highly conserved miRNA families were identified (Additional file 1: Table S4). Meanwhile, 158 novel miRNAs, which had not been reported in soybean before, were detected. Novel miRNAs are considered to be young miRNAs that evolved recently, and are expressed weakly. They are likely to be species-specific miRNAs, which are classified as nonconserved miRNAs, as reported from other plants, such as Arabidopsis thaliana [37]. Unlike conserved miRNAs, only a small percent of soybean novel miRNAs (37.74 \%) were encoded by multiple loci, which was in accordance with the previous results [43].

Previous studies have demonstrated that many miRNAs exist in male-sterile plants. Shen et al. [23] isolated seven novel miRNA families and one known miRNA from a mixture of anthers from a maize CMS line and its maintainer. In 2013, 100 known miRNAs and 81 novel miRNAs of pollen miRNAs from a maize $S$ type CMS line and its fertility-restored line were identified by $\mathrm{Yu}$ et al. [27], with nine of the known miRNAs having significant expression differences between the two lines. In the same year, Yang et al. [26] identified 197 known and 78 new candidate miRNAs during the reproductive development of Brassica juncea, and discovered 47 differentially expressed miRNAs between the CMS and its maintainer. Based on high-throughput sequencing, Wei et al. [28] compared the expression patterns of miRNAs during cotton anther development between the genetic male-sterile mutant and its wild type. A total of 16 conserved miRNA families were identified; six of them were significantly differentially expressed [28]. Recently, Jiang et al. [25] constructed two small RNA libraries from the flower buds of male-sterile and male fertile lines in Brassica campestris ssp. chinensis, and 18 differentially expressed miRNAs with more than two-fold relative changes were identified. In our study, 560 known miRNAs, 105 novel miRNA-3p/miRNA-5p of known premiRNAs, 23 new miRNA members and 159 novel miRNAs (Table 2) in the soybean flower buds of the NJCMS1A and NJCMS1B were identified. We speculate that these miRNAs may participate in the complex network regulating the pollen development process of soybean.

\section{Degradome analysis of the interactions of miRNAs and their targets}

miRNAs regulate genes by mediating gene expression at the post-transcriptional level in plants mainly through mRNA cleavage at certain sites [44]. Currently, bioinformatics-based calculations are commonly used to predict miRNA targets. In recent years, modified $5^{\prime}$ RACE has been frequently used to demonstrate miRNA targets in pollen development [36]. The confirmation procedures were convincing but not effective in identifying a number of candidate targets. Fortunately, a highthroughput method combining 5' RACE with nextgeneration sequencing technology was developed, which has become the new method for identifying targets [45].

With our degradome analysis, 859 targets were chosen and predicted to be cleaved by 297 miRNAs (Additional file 1: Table S9). Among the identified targets of miRNAs, some were previously shown to be involved in floral organ or anther development, such as squamosa 
promoter-binding protein-like [33], APETALA [46], GAMYB or GAMYB-like genes [47], GRAS gene family [48], and auxin response factor [49-51]. The expression levels of squamosa promoter-binding protein-like, APETALA2, GAMYB or GAMYB-like genes and their miRNAs were compared by qRT-PCR (Fig. 6), which showed the inverse relationship between target gene expression and the level of corresponding miRNA. A set of PPR proteins are targeted by gma-miR156, gma-miR160, gma-miR319a-5p, gma-miR4413, gma-miR5674, gmamiR5770 and N-gma-miR164r-3p, which are a group of RNA-binding proteins classified as one of the most important restorer gene families in plants [3]. The transcription of PPR proteins is likely to be inhibited in pollen, so we speculated that these types of miRNAs have correlations with restorer genes that lead to fertility restoration. Some of the targets have not been shown to be associated with pollen development as miRNA targets, such as homeobox-leucine zipper protein and no apical meristem protein. Chen et al. [3] indicated that several models, such as the MADS-box transcription factor in the retrograde regulation model, programmed cell death (PCD) and reactive oxygen species (ROS) in the aberrant PCD model, and ATP and NADH in the energy deficiency model etc. involved mechanisms that may lead to male sterility in plants.

\section{Targets of miRNAs with retrograde regulation and PCD}

Three targets of gma-miR156b and gma-miR156f are members of the MADS-box transcription factor family, which participate in reproductive developmental control and flower development in plants [52, 53]. MADS-box genes are differentially expressed during early germination of the male gametophyte in Nicotiana tabacum [54] and are distinct in the male organ primordia of Arabidopsis thaliana [55]. In maize, their products accumulate in apoptotic bodies during anther dehiscence [56]. Also, MADS-box genes may be in accord with CMS, because in carrot, mitochondria affect the expression of MADS-box genes homologous to GLOBOSA and DEFICIENS, which led to stamens being replaced by carpels, becoming the "carpeloid" CMS type [57]. Yang et al. [58] found that MADS-box genes were associated with cytoplasmic homeosis in CMS stem mustards, by specifically inhibiting the mitochondrial electron transport chain of Brassica juncea. Recently, Huang et al. [59] found a MADS-box transcription factor in soybean using a microarray analysis, and its constitutive expression in tobacco caused sterility because of shortened and curly stalks, and the failure of pollen release from the anthers. However, it was only targeted by gma-miR $156 \mathrm{~b}$ and gma-miR156f in NJCMS1A and was down-regulated (Additional file 2: Figure S8). Even so, we speculated it was highly expressed during the pollen development stage, which would lead to pollen abortion. We may be able to control the expression of gma-miR156b and gma-miR156f to regulate male sterility, therefore the relationship between gma-miR156b and gma-miR156f, and male sterility of the soybean CMS line, needs further research.

The degradome analysis showed that LAC was targeted by gma-miR397a and gma-miR397b-5p. Interestingly, LAC was only targeted by gma-miR397 in NJCMS1B, and the LAC expression level was higher in NJCMS1A than in NJCMS1B (Fig. 6), which may have been caused by the lower expression of gma-miR397 (Additional file 1: Table S8-2) and the negative regulation of LAC expression in NJCMS1A. Some studies have indicated that LAC participates in brassinosteroidregulated plant growth and that overexpressed OsLAC could lead to a semi-sterile phenotype [60]. Brassinosteroids are essential for male fertility in plants, and mutations in brassinosteroid biosynthesis lead to reduced pollen levels, viability, and release efficiencies [61]. Thus, we speculated that gma-miR397 and LAC have some relationship with CMS. However, qRT-PCR showed that gma-miR397 was more highly expressed in NJCMS1A (Figs. 3 and 4), which was inconsistent with the sequencing results and probably related to the anther's developmental period. In maize, miR397 was more highly expressed in the tetrad stage and then rapidly declined in the mononuclear stage [23]. Thus, the relationship of miR397 and LAC with CMS requires further studies.

Novel_mir_006-3p was a novel miRNA found to target the mitochondrial heat shock $70 \mathrm{kDa}$ protein $(\mathrm{mtHsp} 70)$, which influences heat- and $\mathrm{H}_{2} \mathrm{O}_{2}$-induced PCD in plants [62]. Plant PCD, triggered by the retrograde signals from the release of cytochrome $\mathrm{c}$ from mitochondria into the cytosol and the overproduction of ROS, controls cellular degeneration of the tapetum and leads to male sterility [59]. mtHsp70 functions as a motor and is required for polypeptide translocation across the mitochondrial inner membrane subsequent to protein folding reactions in the matrix [63]. In animals, Hsp70-2 participates in synaptonemal complex functions during meiosis in male germ cells and results in male infertility in mice [64]. The results of Chen et al. [65] indicate that Hsp70 has a close relationship with the CMS of sorghum. A lack of Hsp70 expression leads to functional anomalies and decreases the numbers of mitochondria, which can eventually lead to pollen sterility [65]. The novel_mir_006-3p was only found in CMS, and mtHsp70 was downregulated in NJCMS1A (Fig. 6). However, whether opposed expression levels of novel_mir_006-3p and its target gene in the CMS anthers leads to a significant increase in PCD remains to be studied. In addition, a heat shock cognate $70 \mathrm{kDa}$ protein-like gene that was only targeted by gma-miR393a showed a down-regulated 
expression level in NJCMS1A (Additional file 2: Figure S8), which may also be related to male sterility.

\section{Targets of miRNAs involved in responses to oxyradical stress}

NADP-dependent isocitrate dehydrogenase (NADP$\mathrm{ICDH}$ ) and 6-phosphogluconate dehydrogenase were targeted by gma-miR162a, gma-miR162b and gmamiR162c, and gma-miR399d, gma-miR399e, gmamiR399f and gma-miR399g, respectively. Both are NADPH-generating dehydrogenases in plants, and NADPH is a key cofactor in the cellular redox homeostasis and necessary in the metabolism of ROS [66]. In Arabidopsis thaliana, NADP-ICDH activity is regulated by molecules involved in $\mathrm{ROS}$, including $\mathrm{H}_{2} \mathrm{O}_{2}$ [67]. These two types of miRNAs had no significant differences in expression levels between the NJCMS1A and NJCMS1B. Surprisingly, they were only targeted in NJCMS1A and the qRT-PCR results show that NADP$\mathrm{ICDH}$ and 6-phosphogluconate dehydrogenase were down-regulated (Additional file 2: Figure S8). The decreased NADPH level regulated by miRNA in the NJCMS1A may lead to a transient oxidative burst and significant ROS accumulation. However, more studies are needed to understand the relationship between miRNA targeting and ROS that may lead to male sterility in soybean.

Alcohol dehydrogenase 1 (adh1) was the only target of gma-miR2119 which encoding an important enzyme during the conversion of acetaldehyde into ethanol, and regenerating $\mathrm{NAD}^{+}$in the process [68]. In higher plants, the adh gene expression increases dramatically in response to hypoxia [68]. Garabagi et al. [68] showed that the adh1 expressed in the plants appears to be restricted to immature pollen grains. Hajós-Novák et al.'s results revealed that reduced $\mathrm{ADH}$ enzyme activity in the pollen grains would affect the gametophyte [69]. Both sequencing and qRT-PCR indicated that gma-miR2119 expression was up-regulated in NJCMS1A. In contrast, adh1 expression was down-regulated in NJCMS1A (Fig. 6). This may indicate that gma-miR2119 and adh1 are related to pollen development, and thus further research is needed.

\section{Targets of miRNAs in energy metabolism}

NADH-ubiquinone oxidoreductase $24 \mathrm{kDa}$ subunit was identified as the target of gma-miR156b and gmamiR156f, and has a regulatory role in the complex I function in the electron transport chain, which transfers electrons from NADH to ubiquinone through the intramolecular redox centers (FMN and Fe-S centers) in mitochondria [70]. Dihydrolipoyl dehydrogenase (E3) is an important part of the pyruvate dehydrogenase complex, and it was the target of gma-miR169t. Selinski et al. [71] demonstrated that the $\mathrm{ATP} / \mathrm{NAD}(\mathrm{P}) \mathrm{H}$ ratio (by malate valves and $\mathrm{NAD}^{+}$biosynthesis) contributes to satisfying the energy demand during pollen development. In this process, the pyruvate dehydrogenase complex is the critical pathway that supports energy generation for pollen and pollen tube growth [71].

The mitochondrial-like ATP synthase subunit beta was targeted by gma-miR1521a. It is one part of the FATPases (F1F0-ATPases), which are located in mitochondrial plasma membranes and are the prime producers of ATP, using the proton gradient generated by oxidative phosphorylation [72, 73]. One of the targets of gma-miR172c, gma-miR172d and gma-miR172e was the mitochondrial transcription termination factor family protein (mTERFs; homolog of AT4G09620). It was predominantly encoded by the nuclear genome and imported into mitochondria after protein synthesis to play important roles in regulating the organellar transcription machinery [74]. In Arabidopsis thaliana, mutated mTERF15 led to defective mitochondrial functioning and significantly disturbed normal plant vegetative to reproductive growth [74]. These represent core parts of ATPases or mitochondria that produce ATP for pollen development and miRNA biogenesis [26]. These types of genes were only targeted by miRNA (or the target site matched the filter $P<0.05$ ) in NJCMS1A, indicating that there is a close connection between ATP and CMS. qRT-PCR showed that these targets were down-regulated in NJCMS1A (Additional file 2: Figure S8). However, whether an insufficient supply of ATP may lead to male sterility still needs to be researched. The identification of miRNAs and their targets indicates that there are relationships between miRNAs and pollen development. In addition, some miRNA interactions and target gene expressions may only function during a certain period. Thus, in the future we will research the expression of some important miRNAs and their genes in a specific time period to understand the underlying mechanisms that lead to male sterility in the CMS line of soybean.

\section{Conclusion}

In this study, a large number of miRNAs were identified during pollen development in the soybean CMS line NJCMS1A and its maintainer NJCMS1B, by deep sequencing, including 105 novel miRNAs on the other arm of known pre-miRNAs, 23 new miRNA members of known miRNAs, 158 novel miRNAs and 160 highconfidence miRNAs. Additionally, a degradome analysis was used to identify the targets of the discovered miRNAs. Through the expression of miRNAs associated with their targets, we found that there were interactions between miRNAs and their targets. These results revealed that the miRNAs might participate in flower and 
pollen development, which would provide valuable clues for exploring miRNA-mediated regulatory networks in soybean CMS lines.

\section{Methods \\ Plant materials, sample collection and total RNA extraction}

The soybean cytoplasmic male-sterile line NJCMS1A was developed through consecutive backcross procedures with the cultivar N8855 as donor parent and N2899 (designated as NJCMS1B afterwards) as recurrent parent $[8,9,75,76]$. NJCMS1A and NJCMS1B were planted in the summer of 2013 at Jiangpu Experimental Station, National Center for Soybean Improvement, Nanjing Agricultural University, Nanjing, Jiangsu, China. The male-sterile plants were identified through three kinds of methods including the dehiscence of anthers, germination rate of pollens, and performance of plants at maturity. Cytological observation showed that the male abortion of NJCMS1A occurred mainly at the early binucleate pollen stage [77]. Because it is very difficult to judge the precise development stage of pollen from the appearance of the flower buds in soybean, so during the flowering period, the flower buds of different sizes up to and including the abortion stage were collected and mixed from NJCMS1A and NJCMS1B plants respectively, then immediately frozen in liquid nitrogen and stored at $-80{ }^{\circ} \mathrm{C}$ for further use. Total RNA from the flower buds of NJCMS1A and NJCMS1B was extracted using Trizol Reagent (Invitrogen, Carlsbad, CA, USA) according to the manufacturer's protocol.

\section{Small RNA sequencing library construction}

Two small RNA libraries were constructed and sequenced by the Beijing Genomics Institute (BGI, Shenzhen, Guangdong Province, China) using the Illumina HiSeq 2000 System (TruSeq SBS KIT-HS V3, Illumina, Santa Clara, CA, USA). The general process is as follows: first, polyacrylamide gel electrophoresis (PAGE) purification of the RNA bands corresponding to 18-30nt size fractionation was performed to recover small RNAs; second, ligation of the $5 p$ and $3 p$ adapters to the RNA occurred in two steps, each followed by PAGE purification; third, RT-PCR amplification was performed to generate a cDNA colony template library and then purified by PAGE; and fourth, the template was analyzed using an Agilent 2100 Bioanalyzer (Agilent RNA 6000 Nano Kit) and qPCR was performed to produce a qualified library for sequencing.

\section{Bioinformatics analysis of miRNA identification}

After sequencing, raw sequencing reads were processed into clean reads by filtering out adaptor contaminants, oversized insertions, low-quality reads, poly A tags and small tags $<18 \mathrm{nt}$. The small RNA tags were mapped to the soybean genome (ftp://ftp.jgi-psf.org/pub/compgen /phytozome/v9.0/Gmax/assembly/Gmax_189.fa.gz) using SOAP to analyze their expression and distribution on the genome. All small RNA sequences were used to query the RNA sequences for deposited repeats (RepeatMasker output) in GenBank (NCBI GenBank). Rfam (V 11.0) was used to separate the small RNAs that matched rRNA, scRNA, snoRNA, snRNA, tRNA and remove the degraded mRNA fragments (ftp://ftp.jgi-psf.org/pub/compgen/phytozome/v9.0/Gmax/annotation/Gmax_189_gene.gff3.gz) in the small RNA tags that aligned to exons and introns. Next, to identify conserved miRNAs, clean reads were compared with known miRNAs of soybean deposited at miRBase 21.0 (http://www.mirbase.org/). Finally, the other sequences that did not map to known miRNAs and other kinds of small RNA were referred to as unannotated sequences for new miRNA member identification and novel miRNA prediction.

We identified potentially novel miRNAs using MIREAP (https://source-forge.net/projects/ mireap/), and the secondary structure was predicted by the mfold Web server (http://mfold.rna.albany.edu/?q=mfold/RNA-Folding-Form) [78]. For novel miRNAs, the number of mature miRNAs with predicted hairpins must be no fewer than five in the alignment results (if the miRNA-3p and miRNA-5p are simultaneously on the two arms of premiRNA secondary structures, then one of the mature miRNAs must be no fewer than five). The criteria used for selecting miRNAs must meet the following characteristics based on Meyers et al. [32], Zhang et al. [79], and Kozomara et al. [33]: (1) The candidate miRNA-5p and miRNA-3p are derived from opposite stem-arms with minimal matched nucleotide pairs exceeding $16 \mathrm{nt}$ and with maximal size differences up to $4 \mathrm{nt}$; (2) The most abundant reads from each arm of the precursor must pair in the mature miRNA duplex with a 2-nt, 3' overhang; (3) The number of asymmetric bulges within the miRNA-5p/miRNA-3p duplex must be fewer than one, and the size of the asymmetric bulges must be fewer than two bases; (4) The candidate miRNA precursor must have high negative MFE and MFEI, with MFE $<-0.2 \mathrm{kcal} / \mathrm{mol} / \mathrm{nt}$ and MFEI $>0.85$. Then, the sequence is most likely a miRNA.

The frequency of miRNAs in the two libraries was normalized to the expression of transcript per million (TPM; normalized expression $=$ Actual miRNA count $\times 1 \quad 000$ 000/Total clean read counts). Following normalization, if one of the miRNA gene expressions between the two samples was zero, then it was revised to 0.01 , and if the miRNA gene expression of two samples was less than 1 , owing to their too low expression levels, then they were not included in the differential expression analysis. The differential expressions of miRNAs were calculated using 
fold changes and $P$-values from the normalized expression with the selection threshold of $\mid \log _{2} \mathrm{FC}$ (Fold Change) $|\geq|$ $\pm 1 \mid$ and $P$-value $<0.05$, respectively.

\section{Degradome library construction, data analysis and target identification}

Two degradome libraries were constructed according to the methods by Addo-Quage et al. and German et al. [14, 80]. In brief, poly-A-containing mRNA was purified from total RNA, and then a 5' RNA adaptor containing a MmeI recognition site was ligated to the $5^{\prime}$-phosphate of the poly-A RNA. Subsequently, reverse transcription was performed to generate first-strand cDNA, followed by digestion with $M m e \mathrm{I}$ and ligation to a 3' adaptor. Finally, the ligation products were amplified using PCR, gel-purified and subjected to deep sequencing on the Illumina HiSeq 2000 (TruSeq SBS KIT-HS V3, Illumina; BGI, Shenzhen, Guangdong Province, China).

Data filtering was performed to remove adaptor sequences and/or low-quality reads present in the raw reads, resulting in clean reads. This was followed by the collection of 20-21-nt sequences of high quality for subsequent analysis. The degradome tags were mapped to the soybean genome using SOAP and then annotated with rRNA, scRNA, snoRNA, snRNA and tRNA from Rfam and GenBank to separate matched tags from unannotated tags. Degraded sequences with over $70 \%$ single bases in the clean reads were identified as poly-N, which were not used to predict subsequent degradation sites. The statistics of cleaved sites and T-plots were processed by CleaveLand 3.0 (http:// axtell-lab-psu.weebly.com/cleaveland.html), and PAREsnip (http://srna-workbench.cmp.uea.ac.uk/tools/paresnip/) was used for the prediction of miRNA targets and hypothesis testing.

The tags mapped to cDNA_sense were used to predict cleavage sites. Categorization of degradome peaks was performed as follows: The height of the degradome peak at each occupied transcript position was placed into one of five possible categories, described below. Category 0: $>1$ raw read at the position, abundance at the position is equal to the maximum on the transcript, and there is only one maximum on the transcript; Category 1: > 1 raw read at the position, abundance at the position is equal to the maximum on the transcript, and there is more than one maximum position on the transcript; Category 2: $>1$ raw read at the position, abundance at the position is less than maximum but higher than the median for the transcript; Category 3: $>1$ raw read at the position, abundance at the position is equal to or less than the median for the transcript; and Category 4: only 1 raw read at the position.
Alignments with scores up to 4.5 , where G:U pairs scored 0.5 , and no mismatches were found at the site between the $10^{\text {th }}$ and $11^{\text {th }}$ nucleotides of the corresponding miRNAs, were considered potential targets.

\section{Quantitative real-time PCR validation}

Stem-loop qRT-PCR and qRT-PCR were carried out to validate differential expressional levels of miRNAs and miRNA targets, respectively [81]. All primers (Additional file 1: Table S11) were designed based on the mature miRNA and mRNA sequences, and synthesized commercially (Invitrogen, Shanghai, China). According to the procedures provided by the iScript Select cDNA Synthesis Kit (containing GSP enhancer solution, BIO-RAD, USA), $1 \mu \mathrm{g}$ of total RNA was reverse-transcribed by iScript reverse transcriptase using stem-loop primers. A qRT-PCR analysis was carried out using iTaq Universal SYBR Green Supermix (BIO-RAD, USA) on the Bio-Rad CFX96 machine (CFX96 Touch, BIO-RAD, USA). All reactions were run with three biological replicates, and gma-miR1520d was used as the internal control gene [82]. The miRNA relative expression levels were quantified using the $2^{-\Delta \Delta \mathrm{Ct}}$ method. Student's $t$-test was performed to compare mRNA differences in expression between NJCMS1A and NJCMS1B. The means of $2^{-\Delta \Delta \mathrm{Ct}}$ were considered significantly different at $P<0.05$.

\section{Online data deposition}

All of the small RNA-seq and degradome-seq data were submitted to the National Center for Biotechnology Information (NCBI) under the accession number PRJNA304685.

\section{Additional files}

\begin{abstract}
Additional file 1: Table S1. Summary of small RNA annotations from NJCMS1A and NJCMS1B. Table S2. Known miRNAs identified in NJCMS1A and NJCMS1B. Table S3. Family member distribution in conserved miRNA families. Table S4. Summary of miRNA families found in NJCMS1A and NJCMS1B. Table S5. Novel miRNAs on the other arm of known pre-miRNAs. Table S6. Novel miRNAs identified in NJCMS1A and NJCMS1B. Table S7-1. High-confidence known miRNAs identified in NJCMS1A and NJCMS1B. Table S7-2. High-confidence novel miRNAs identified in NJCMS1A and NJCMS1B. Table S8-1. The up-regulated miRNAs identified in NJCMS1A and NJCMS1B. Table S8-2. The down-regulated miRNAs identified in NJCMS1A and NJCMS1B. Table S9. The targets of miRNAs identified in NJCMS1A and NJCMS1B. Table S10. Targets of novel miRNAs in NJCMS1A and NJCMS1B. Table S11. Primers used in this study. (ZIP 637 kb)

Additional file 2: Figure S1. Known miRNA distribution in NJCMS1A and NJCMS1B. Figure S2. Family member distribution in conserved miRNA families in NJCMS1A and NJCMS1B. Figure S3. Predicted secondary structures of novel miRNAs on the other arm of known pre-miRNAs. Figure S4. Predicted secondary structures of new miRNA members. Figure S5. Predicted secondary structures of novel miRNAs. Figure S6. Predicted secondary structures of high-confidence miRNAs. Figure S7. GO analysis of miRNA targets. Figure S8. Expression levels of miRNA targets in NJCMS1A and NJCMS1B. (ZIP $4771 \mathrm{~kb}$ )
\end{abstract}

\section{Abbreviations}

CMS: Cytoplasmic male sterility; miRNA: microRNA; MFE: Minimum free energies; MFEl: Minimal folding energy indices; qRT-PCR: Quantitative real- 
time PCR; SPL: Squamosa promoter-binding protein-like; AP2: APETALA2; PPR: Pentatricopeptide repeat-containing; Hsp70: Heat shock 70 kDa protein; LAC: Laccase; GO: Gene Ontology; PCD: Programmed cell death; ROS: Reactive oxygen species; mtHsp70: Heat shock 70 kDa protein, mitochondrial; NADP-ICDH: NADP-dependent isocitrate dehydrogenase; ADH: Alcohol dehydrogenase; mTERFs: Mitochondrial transcription termination factor family protein.

\section{Competing interests}

The authors declare that they have no competing interests.

\section{Authors' contributions}

SPY, JYG and XLD conceived and designed the experiments. XLD, JJL, HZ, $\Pi \mathrm{TH}, \mathrm{SHH}, \mathrm{YWL}$ performed the experiments. XLD analyzed the data. XLD, Jال contributed reagents/materials/analysis tools. XLD, $\mathrm{HZ}$ and $\mathrm{SHH}$ conceived the qRT-PCR experiments and analyzed the data. XLD, SPY conceived the experiments and wrote the manuscript. All authors read and approved the final manuscript.

\section{Acknowledgements}

This work was supported by the National Hightech R \& D Program of China (2011AA10A105), the National Transgene Science and Technology Major Program of China (2011ZX08004-005, 2013ZX08004-005, 2014ZX08004-005), the National Key Basic Research Program of China (2011CB109301), the Program for Changjiang Scholars and Innovative Research Team in University (PCSIRT13073), and Jiangsu Collaborative Innovation Center for Modern Crop Production (JCIC-MCP).

\section{Author details}

All authors came from Soybean Research Institute, National Center for Soybean Improvement, MOA Key Laboratory of Biology and Genetic Improvement of Soybean (General), State Key Laboratory of Crop Genetics and Germplasm Enhancement, Nanjing Agricultural University, Nanjing, 210095, Jiangsu, People's Republic of China.

Received: 11 July 2015 Accepted: 21 December 2015

Published online: 05 January 2016

\section{References}

1. Hanson MR. Plant mitochondrial mutations and male sterility. Annu Rev Genet. 1991;25:461-86

2. Hanson MR, Bentolila S. Interactions of mitochondrial and nuclear genes that affect male gametophyte development. Plant Cell. 2004;16:S154-69.

3. Chen LT, Liu YG. Male sterility and fertility restoration in crops. Annu Rev Plant Biol. 2014:65:579-606.

4. Hu J, Wang K, Huang W, Liu G, Gao Y, Wang J, et al. The rice pentatricopeptide repeat protein RF5 restores fertility in Hong-Lian cytoplasmic male-sterile lines via a complex with the glycinerich protein GRP162. Plant Cell. 2012;24:109-22.

5. Horn R, Gupta KJ, Colombo N. Mitochondrion role in molecular basis of cytoplasmic male sterility. Mitochondrion. 2014;19:198-205.

6. Davis WH. Route to hybrid soybean production. United States Patent. 1985; US 4545146

7. Sun H, Zhao LM, Huang M. Studies on cytoplasmic-nuclear male sterile soybean. Chin Sci Bull. 1993;38:1535-1536.

8. Gai JY, Cui ZL, Ji DF, Ren ZJ, Ding DR. A report on the nuclear cytoplasmic male sterility from a cross between two soybean cultivars. Soy Genet Newsl. 1995;22:55-8.

9. Ding DR, Gai JY, Cui ZL, Yang SP, Qiu JX. Development and verification of the cytoplasmic-nuclear male sterile soybean line NJCMS1A and its maintainer NJCMS1B. Chin Sci Bull. 1998;44:191-92.

10. Bai YN, Gai JY. Development of soybean cytoplasmic-nuclear male-sterile line NJCMS2A and restorability of its male ferlity. Sci Agric Sin. 2003:36:740-45.

11. Jones-Rhoades MW, Bartel DP, Bartel B. MicroRNAs and their regulatory roles in plants. Annu Rev Plant Biol. 2006:57:19-53.

12. Mallory AC, Vaucheret $H$. Functions of microRNAs and related small RNAs in plants. Na Genet. 2006:38:S31-6.

13. Lelandais-Brière C, Sorin C, Declerck M, Benslimane A, Crespi M, Hartmann C. Small RNA diversity in plants and its impact in development. Curr Genomics. 2010;11:14-23.
14. Addo-Quaye C, Eshoo TW, Bartel DP, Axtell MJ. Endogenous siRNA and miRNA targets identified by sequencing of the Arabidopsis degradome. Curr Biol. 2008;18:758-62.

15. Shamimuzzaman M, Vodkin L. Identification of soybean seed developmental stage-specific and tissue-specific miRNA targets by degradome sequencing. BMC Genomics. 2012;13:310.

16. Subramanian S, Fu Y, Sunkar R, Barbazuk WB, Zhu JK, Yu O. Novel and nodulation-regulated microRNAs in soybean roots. BMC Genomics. 2008;9:160

17. Chen $\mathrm{R}, \mathrm{Hu} \mathrm{Z}$, Zhang $\mathrm{H}$. Identification of microRNAs in wild soybean (Glycine soja). J Integr Plant Biol. 2009;51:1071-9.

18. Zeng HQ, Zhu YY, Huang SQ, Yang ZM. Analysis of phosphorus-deficient responsive miRNAs and cis-elements from soybean (Glycine max L.). J Plant Physiol. 2010;167:1289-97.

19. Song QX, Liu YF, Hu XY, Zhang WK, Ma B, Chen SY, et al. Identification of miRNAs and their target genes in developing soybean seeds by deep sequencing. BMC Plant Biol. 2011;11:5

20. Turner M, Yu O, Subramanian S. Genome organization and characteristics of soybean microRNAs. BMC Genomics. 2012;13:169.

21. Hu Z, Jiang QY, Ni ZY, Chen R, Xu S, Zhang H. Analyses of a Glycine max degradome library identify microRNA targets and microRNAs that trigger secondary siRNA biogenesis. J Integr Plant Biol. 2013;55:160-76.

22. Goette W, Liu ZR, Xia J, Zhang WX, Zhao PX, An YQC(Charles). Systems and evolutionary characterization of microRNAs and their underlying regulatory networks in soybean cotyledons. PLoS One. 2014;9:e86153.

23. Shen YO, Zhang ZM, Lin HJ, Liu HL, Chen J, Peng H, et al. Cytoplasmic male sterility-regulated novel microRNAs from maize. Funct Integr Genomics. 2011;11:179-91.

24. Zhou H, Liu QJ, Li J, Jiang DG, Zhou LY, Wu P, et al. Photoperiod- and thermo-sensitive genic male sterility in rice are caused by a point mutation in a novel noncoding RNA that produces a small RNA. Cell Res. 2012;22: 649-60

25. Jiang JX, LV ML, Liang Y, Ma ZM, Cao JS. Identification of novel and conserved miRNAs involved in pollen development in Brassica campestris ssp. chinensis by high-throughput sequencing and degradome analysis. BMC Genomics. 2014;15:146.

26. Yang JH, Liu XY, Xu BC, Zhao N, Yang XD, Zhang MF. Identification of miRNAs and their targets using high-throughput sequencing and degradome analysis in cytoplasmic male-sterile and its maintainer fertile lines of Brassica juncea. BMC Genomics. 2013;14:9.

27. $\mathrm{Yu} \mathrm{JH}$, Zhao $Y X$, Qin $Y T$, Yue B, Zheng $Y L$, Xiao HL. Discovery of microRNAs associated with the $S$ type cytoplasmic male sterility in maize. J Integr Agr 2013;12:229-38

28. Wei MM, Wei HL, Wu M, Song MZ, Zhang JF, Yu JW, et al. Comparative expression profiling of miRNA during anther development in genetic male sterile and wild type cotton. BMC Plant Biol. 2013;13:66.

29. Joshi T, Yan Z, Libault M, Jeong DH, Park S, Green PJ, et al. Prediction of novel miRNAs and associated target genes in Glycine max. BMC Bioinformatics. 2010;11:S14

30. Kulcheski FR, de Oliveira FLV, Molina LG, Almerão MP, Rodrigues FA Marcolino J, et al. Identification of novel soybean microRNAs involved in abiotic and biotic stress. BMC Genomics. 2011:12:307.

31. Zhang BH, Pan XP, Cannon CH, Cobb GP, Anderson TA. Conservation and divergence of plant microRNA genes. Plant J. 2006:46:243-59.

32. Meyers BC, Axtell MJ, Bartel B, Bartel DP, Baulcombe D, Bowman JL, et al. Criteria for annotation of plant microRNAs. Plant Cell. 2008:20:3186-90.

33. Kozomara A, Griffiths-Jones S. miRBase: annotating high confidence microRNAs using deep sequencing data. Nucleic Acids Res. 2014:42:D68-73.

34. Xing SP, Salinas M, Hohmann S, Berndtgen R, Huijser P. miR156-targeted and nontargeted SBP-box transcription factors act in concert to secure male fertility in Arabidopsis. Plant Cell. 2010;22:3935-50.

35. Chambers C, Shuai B. Profiling microRNA expression in Arabidopsis pollen using microRNA array and real-time PCR. BMC Plant Biol. 2009;9:87.

36. Grant-Downton R, Le TG, Schmid R, Rodriguez-Enriquez J, Hafidh S, Mehdi S, et al. MicroRNA and tasiRNA diversity in mature pollen of Arabidopsis thaliana. BMC Genomics. 2009;10:643

37. Rajagopalan R, Vaucheret $H$, Trejo J, Bartel DP. A diverse and evolutionarily fluid set of microRNAs in Arabidopsis thaliana. Genes Dev. 2006;20:3407-25.

38. Kou SJ, Wu XM, Liu Z, Liu YL, Xu Q, Guo WW. Selection and validation of suitable reference genes for miRNA expression normalization by quantitative RT-PCR in citrus somatic embryogenic and adult tissues. Plant Cell Rep. 2012;31:2151-63. 
39. Wang TZ, Chen L, Zhao MG, Tian QY, Zhang WH. Identification of droughtresponsive microRNAs in Medicago truncatula by genome-wide highthroughput sequencing. BMC Genomics. 2011;12:367.

40. Wei LQ, Yan LF, Wang T. Deep sequencing on genome-wide scale reveals the unique composition and expression patterns of microRNAs in developing pollen of Oryza sativa. Genome Biol. 2011;12:R53.

41. Li DT, Wang LW, Liu X, Cui DZ, Chen TT, Zhang H, et al. Deep sequencing of maize small RNAs reveals a diverse set of microRNA in dry and imbibed seeds. PLoS One. 2013;8:e55107.

42. Guo L, Lu ZH. Global expression analysis of miRNA gene cluster and family based on isomiRs from deep sequencing data. Comput Biol Chem. 2010;34:165-71.

43. Ma ZR, Coruh C, Axtell MJ. Arabidopsis lyrata Small RNAs: transient MIRNA and small interfering RNA loci within the Arabidopsis genus. Plant Cell. 2010;22:1090-103.

44. Bartel DP. MicroRNAs: target recognition and regulatory functions. Cell. 2009;136:215-33.

45. Meng YJ, Gou LF, Chen DJ, Wu P, Chen M. High-throughput degradome sequencing can be used to gain insights into microRNA precursor metabolism. J Exp Bot. 2010;61:3833-7.

46. Aukerman MJ, Sakai $\mathrm{H}$. Regulation of flowering time and floral organ identity by a microRNA and its APETALA2-like target genes. Plant Cell. 2003; 15:2730-41.

47. Alonso-Peral MM, Li JY, Li YJ, Allen RS, Schnippenkoetter W, Ohms S, et al. The microRNA159-regulated GAMYB-like genes inhibit growth and promote programmed cell death in Arabidopsis. Plant Physiol. 2010;154:757-71.

48. Llave C, Xie Z, Kasschau KD, Carrington JC. Cleavage of Scarecrow-like mRNA targets directed by a class of Arabidopsis miRNA. Science. 2002:297:2053-6.

49. Yang FX, Liang G, Liu DM, Yu DQ. Arabidopsis miR396 mediates the development of leaves and flowers in transgenic tobacco. J Plant Biol. 2009; 52:475-81.

50. Liang G, He H, Li Y, Wang F, Yu DQ. Molecular mechanism of miR396 mediating pistil development in Arabidopsis thaliana. Plant Physiol. 2014; 164:249-58.

51. Baucher M, Moussawi J, Vandeputte OM, Monteyne D, Mol A, Pérez-Morga D, et al. A role for the miR396/GRF network in specification of organ type during flower development, as supported by ectopic expression of Populus trichocarpa miR396c in transgenic tobacco. Plant Biol. 2013;15:892-8.

52. Alvarez-Buylla ER, Liljegren SJ, Pelaz S, Gold SE, Burgeff C, Ditta GS, et al. MADS-box gene evolution beyond flowers: expression in pollen, endosperm, guard cells, roots and trichomes. Plant J. 2000;24:457-66.

53. Sundström J, Carlsbecker A, Svensson ME, Svenson M, Johanson U, Theissen $G$, et al. MADS-box genes active in developing pollen cones of Norway spruce (Picea abies) are homologous to the B-class floral homeotic genes in angiosperms. Dev Genet. 1999;25:253-66.

54. Steiner C, Bauer J, Amrhein N, Bucher M. Two novel genes are differentially expressed during early germination of the male gametophyte of Nicotiana tabacum. Biochim Biophys Acta. 2003;1625:123-33.

55. Sundström J, Engström P. Conifer reproductive development involves Btype MADS-box genes with distinct and different activities in male organ primordial. Plant J. 2002;31:161-9.

56. Schreiber DN, Bantin J, Dresselhaus T. The MADS box transcription factor ZmMADS2 is required for anther and pollen maturation in maize and accumulates in apoptotic bodies during anther dehiscence. Plant Physiol. 2004;134:1069-79.

57. Linke B, Nothnagel T, Börner T. Flower development in carrot CMS plant: mitochondria affect the expression of MADS box genes homologous to GLOBOSA and DEFICIENS. Plant J. 2003;34:27-37.

58. Yang JH, Qi XH, Zhang MF, Yu JQ. MADS-box genes are associated with cytoplasmic homeosis in cytoplasmic male-sterile stem mustard as partially mimicked by specifically inhibiting mtETC. Plant Growth Regul. 2008:56:191-201.

59. Huang F, Xu GL, Chi YJ, Liu HC, Xue Q, Zhao TJ, et al. A soybean MADS-box protein modulates floral organ numbers, petal identity and sterility. BMC Plant Biol. 2014;14:89.

60. Zhang YC, Yu Y, Wang CY, Li ZY, Liu Q, Xu J, et al. Overexpression of microRNA OsmiR397 improves rice yield by increasing grain size and promoting panicle branching. Nature Biotechnol. 2013;31:848-52.

61. Ye QQ, Zhu WJ, Li L, Zhang SS, Yin YH, Ma H, et al. Brassinosteroids control male fertility by regulating the expression of key genes involved in Arabidopsis anther and pollen development. Proc Natl Acad Sci USA. 2010;107:6100-5.
62. Qi YC, Wang HJ, Zou Y, Liu C, Liu YQ, Wang Y, et al. Over-expression of mitochondrial heat shock protein 70 suppresses programmed cell death in rice. FEBS Letters. 2011;585:231-9.

63. Su PH, Li H. Stromal Hsp70 is important for protein translocation into pea and Arabidopsis chloroplasts. Plant Cell. 2010;22:1516-31.

64. Dix DJ, Allen JW, Collins BW, Mori C, Nakamura N, Poorman-Allen P, et al. Targeted gene disruption of Hsp70-2 results in failed meiosis, germ cell apoptosis, and male infertility. Proc Natl Acad Sci USA. 1996; 93:3264-8.

65. Wang L, Qi XH, Liu GQ, Zhang YM, Chen JN. The relationship between the change of mitochondria and the fertility in CMS line of sorghum after heat shock. Acta Genet Sin. 2000;27:834-8

66. Leterrier M, Barroso JB, Valderrama R, Palma JM, Corpas FJ. NADPdependent isocitrate dehydrogenase from Arabidopsis roots contributes in the mechanism of defence against the nitro-oxidative stress induced by salinity. Scientific World Journal. 2012;2012:694740.

67. Leterrier M, Barroso JB, Palma JM, Corpas FJ. Cytosolic NADP-isocitrate dehydrogenase in Arabidopsis leaves and roots. Biol Plantarum. 2012;56: 705-10.

68. Garabagi F, Strommer J. Distinct genes produce the alcohol dehydrogenases of pollen and maternal tissues in Petunia hybrid. Biochem Genet. 2004:42:199-208.

69. Hajós-Novák M, Nagy AH, Dallmann G. Study of the alcohol dehydrogenase1 (Adh1) gene in tetraploid corn: expression in the pollen grains and restriction fragment length polymorphism. Acta Biol Hung. 1997;48:87-94.

70. Hegde R. The 24-kDa subunit of the bovine mitochondrial NADH: ubiquinone oxidoreductase is a G protein. Biochem Biophys Res Commun. 1998;244:620-9.

71. Selinski J, Scheibe R. Pollen tube growth: where does the energy come from? Plant Signal Behav. 2014;9:12,e977200.

72. Müller V, Cross RL. The evolution of A-, F-, and V-type ATP synthases and ATPases: reversals in function and changes in the $\mathrm{H}^{+} /$ATP coupling ratio. FEBS Lett. 2004:576:1-4.

73. Zhang X, Niwa H, Rappas M. Mechanisms of ATPases-a multi-disciplinary approach. Curr Protein Pept Sci. 2004:5:89-105.

74. Hsu YW, Wang HJ, Hsieh MH, Hsieh HL, Jauh GY. Arabidopsis mTERF15 is required for mitochondrial nad 2 intron 3 splicing and functional complex I activity. PLOS ONE. 2014;9:e112360.

75. Ding DR, Gai JY, Cui ZL, Yang SP, Qiu JX. Development and verification of the cytoplasmic-nuclear male sterile soybean line NJCMS1A and its maintainer NJCMS1B. Chin Sci Bull. 1999:44:191-2.

76. Ding DR, Gai JY, Cui ZL, Qiu JX. Development of a cytoplasmic-nuclear male-sterile line of soybean. Euphytica. 2002;124:85-91.

77. Fan JM. Studies on cyto-morphological and cyto-chemical features of cytoplasmic-nuclear male-sterile lines of soybeans (Glycine max (L.) Merr.). M. Sc. Thesis. Nanjing: Nanjing Agricultural University press. 2003.

78. Zuker M. Mfold web server for nucleic acid folding and hybridization prediction. Nucleic Acids Res. 2003;31:3406-15.

79. Zhang BH, Pan XP, Cox SB, Cobb GP, Anderson TA. Evidence that miRNAs are different from other RNAs. Cell Mol Life Sci. 2006:63:246-54.

80. German MA, Pillay M, Jeong DH, Hetawal A, Luo S, Janardhanan P, et al. Global identification of microRNA-target RNA pairs by parallel analysis of RNA ends. Nature Biotechnol. 2008:26:941-6.

81. Chen C, Ridzon DA, Broomer AJ, Zhou Z, Lee DH, Nguyen JT, et al. Realtime quantification of microRNAs by stem-loop RT-PCR. Nucleic Acids Res. 2005; 33:e179.

82. Kulcheski FR, Marcelino-Guimaraes FC, Nepomuceno AL, Abdelnoor RV Margis $\mathrm{R}$. The use of microRNAs as reference genes for quantitative polymerase chain reaction in soybean. Anal Biochem. 2010;406:185-92. 